\title{
Personalisierte Psychiatrie ist mehr als Präzisionspsychiatrie
}

„Hat doch offenbar auch der Arzt nicht die „Gesundheit-an-sich“ im Auge, sondern die des Menschen, vielmehr die seines Patienten. Denn seine Kunst gilt dem Einzelnen.“ Aristoteles [1]

„Personalized medicine (...) requires us to know the parameters that define the individuality of the particular patient."

O. Wolkenhauer et al. [2]

\section{Mehr Präzision, bitte!}

Die Forderung nach einer „personalisierten Psychiatrie“ ist in vieler Munde. Sie folgt aus der Erfahrung, dass das Programm der evidenzbasierten Psychiatrie weit hinter den Erwartungen zurückgeblieben ist: Trotz großem Forschungsaufwand leisten die vorhandenen Möglichkeiten an Diagnostik, Therapie und wissenschaftlicher Durchdringung bei weitem nicht das, was wir noch vor Kurzem erwartet haben.

Ein Grund für die Erfolglosigkeit der evidenzbasierten Psychiatrie ist die Orientierung an einer invaliden Krankheitslehre: Die kanonisierten Typen psychischer Störungen, die ICD-10 oder DSM-5 ausweisen, sind nicht durch bekannte ätiologische oder pathologische Bedingungen begründet. Es handelt sich um Syndrom-Definitionen, die aus dem Bemühen, „signifikante Gestalten“ im Flux der Phänomene zu erkennen, resultierten, heute aber weder klinisch-deskriptiven noch wissenschaftlich-explanatorischen Ansprüchen genügen.

Der Grund für die schlechte Passung zwischen Syndrom-Definition und Patient ist unschwer zu erkennen: Das zu beurteilende, psychisch kranke Individuum ist ein dynamisches, sich unter gegebenen Bedingungen selbst organisierendes System, dessen „psychopathologischer Phänotyp“ in jeder Dimension variieren kann und der vom potenziellen Einfluss jeder Ebene abhängt - von der Genexpression bis zur sozialen Lage. Dies erklärt auf der klinischen Ebene, warum Komorbidität, Syndrom- wechsel und Therapieversagen die Regel und nicht die Ausnahme sind. Und es erklärt auf theoretischer Ebene, warum das „medizinische Modell“ psychischer Störungen, das eine störungsspezifische „gemeinsamen pathogenen Ursache“ unterstellt, empirisch erfolglos bleibt: Wir kennen nicht einen einzigen biologischen Marker, der einen bestimmten Typ psychischer Störung auf spezifische Weise von anderen Störungen bzw. vom Spektrum des Normalen unterscheidet [3].

Angesichts dieser Schwierigkeiten erscheint der Gedanke, dass das Störungsgeschehen ein besonderes, ein individuelles sein könnte, anstatt, wie wir bisher glaubten, ein allgemeines, ein störungsspezifisches zu sein, erfrischend: Wir sollten, folgern führende Forscher, deswegen homogenere Gruppen von Exemplaren eines möglichst scharf umrissenen (Teil-)Phänotyps in Bezug auf alle erdenklichen pathogenen Domänen untersuchen. (Beispielhaft dafür ist der Research Domain Criteria-Ansatz des NIMH). Erklärtes Ziel dieser Bemühungen ist es, sich dem tatsächlichen Bedingungsgefüge durch eine individualisierende Betrachtungsweise anzunähern. Dadurch sollte es nicht nur möglich werden, endlich valide Zusammenhänge zwischen psychopathologischen und organismischen Parametern zu etablieren, sondern auch effektivere, individuell zugeschnittene Therapien anzubieten.

Diesen Überlegungen folgend wird personalisierte Psychiatrie von vielen als „Präzisionspsychiatrie“ verstanden: Es geht um die möglichst präzise Erfassung der pathogenen biologischen Bedingungen im konkreten Einzelfall. Große Hoffnungen werden dabei auf die sich schnell entwickelnden technologischen Möglichkeiten der molekularen Omics-Medizin gesetzt: Diese verspricht, die Bedingungen zu erhellen, die auf Zellebene (z. B. als Genom, Exom, Transkriptom, Metabolom), auf Organebene (etwa als spezifisches Konnektom oder dessen „Kognom“) oder auf Organismusebene (z. B. als Antikörperbiom, Mikrobiom) zum Zustandekommen eines individuellen Störungsbildes beitragen. Um den Schatz der nach dieser Logik generierten, immensen Datenmassen therapiedienlich heben zu können, müssen Muster erkannt und mittels Vergleich mit anderen Patientendaten interpretiert werden. Dies dürfte am ehesten in der Form individueller pathogenen Systemdynamiken gelingen [4].

\section{Der Patient als Person}

Ich werde im Folgenden etwas Wasser in den süßen Wein der präzisionspsychiatrischen Hoffnungen gießen: Denn die Gleichsetzung von „personalisierter Psychiatrie“ mit „Präzisionspsychiatrie“ erscheint mir bedenklich. Personalisierte Psychiatrie ist mehr als nur Präzisionsdiagnostik im Sinne einer möglichst „exakten Erfassung des biomolekularen Geschehens im Organismus “ bzw. als Präzisionstherapie im Sinne einer möglichst „präzisen Korrektur individueller pathogener Prozesse im Organismus“. Denn personalisiert heißt: der Person entsprechend - der ganzen Person und nicht etwa nur dem sub-personalen biologischen Geschehen, das diese Person ausmacht. Der Patient ist ein Organismus aus Fleisch und Blut, dessen sozial eingebettete Omics-Dynamik ein Seelenleben hervorbringt. Bewusstsein, subjektive Wahrnehmungs-, Erlebens- und Handlungsperspektive, ein „Netz“ aus individuellen Bedeutungen, Präferenzen, Narrativen und Werte sind Merkmale, die Personen kennzeichnen [5]. Wir können, um ein Beispiel zu bemühen, den depressiven Patienten Müller nicht verstehen, ohne um die Liebe zu seiner verstorbenen Frau, sein Festhalten an gemeinsamen Plänen und seinen Ärger über Bayern München zu wissen. Psychische Störungen, daran müssen Omics-Begeisterte gelegentlich erinnert werden, sind Störungen des Erlebens und Handelns, die sich auf der personalen Ebene manifestieren. Psychische Störungen, die allein anhand sub-personaler Merkmale diagnostiziert werden könnten, gibt es nicht. 
Zur Verdeutlichung mag es hilfreich sein, an die personale Dimension der Psychopathologie zu erinnern: Deskriptoren wie „gedrückte Stimmung“, „Ängste“, „Denkstörungen“ oder „soziale Interaktionsprobleme“ beziehen sich auf misslingende Aktivitäten von Personen. Stimmungen etwa sind Ausdruck des individuellen Sich-inder-Welt-Erlebens; Ängste sind ohne den intentionalen Gehalt, den sie für den Betroffenen haben, nicht zu verstehen; Denkstörungen lassen sich nur als Abweichungen von konventionellen Diskursstandards erfassen; und unzureichende Selbststeuerung oder unangemessenes Interaktionsverhalten werden anhand von sozialen Stereotypen situativ angemessenen Handelns beurteilt. Kurz: Wir können Störungen der Psyche nur auf dem Hintergrund der lebensweltlichen Normen erfassen, die für das (Selbst-)Verständnis von Personen konstitutiv sind, ganz gleich auf welcher Ebene „die eigentlichen Ursachen “ dieser Störungen angesiedelt sein mögen. Psychopathologie - als Grundlagendisziplin der Psychiatrie - ist Personenpsychologie mit Blick auf die Defizite personalen Handelns. Sie gründet in unserem kollektiven Selbstverständnis als rationalen Personen.

\section{Die Person als Organismus}

Trotz bereitwilliger Lippenbekenntnisse zugunsten eines systembiologischen Verständnisses bleiben viele Vertreter der Präzisionspsychiatrie einem mechanistischen Denken verhaftet. Deswegen sei betont: Organismen sind keine Maschinen, keine Aggregate von Mechanismen, deren Wechselwirkungen die Phänomene erklären. Der neuerdings immer beliebter werdende Verweis auf die Ebene „mechanistischer Erklärungen “ ist nur metaphorisch zu verstehen: Er meint, dass die Zusammenhänge sich verhalten, als wäre ein Mechanismus im Spiel. Dabei arbeiten die unterstellten „bio-pathogenen Mechanismen“ anders, als gedacht, wie die folgenden 2 Überlegungen zeigen mögen.

Erstens, biologische Prozesse sind nie stabil oder passiv wie Zahnräder, die auf das Einrasten des benachbarten Zahnrads warten. Vielmehr operieren alle beteiligten Elemente stets aktiv: Sie interagieren in selbst organisierten Netzwerken und sind zudem in ständigem Stoffaustausch begriffen - also instabil. (Der stabilste Teil des menschlichen Körpers ist das Skelett, dass im Verlauf von etwa 10 Jahre vollständig ersetzt wird.) Dies ist Folge der „interaktiven Dialektik des Lebens“: Lebewesen müssen „geschlossen“ operieren, um ihre interne Organisation aufrecht zu erhalten. Und sie müssen sich „offen“ zeigen, um mittels Austausch von Materie und Energie einen Zustand „fern des thermodynamischen Gleichgewichts“ zu gewährleisten. Die „Mechanismen“, die viele Autoren dabei am Werke sehen und in Lehrbuchdarstellungen festhalten, sind Ausdruck einer „Schnappschuss-Ontologie“, die dem tatsächlichen, prozesshaften Geschehen nicht entspricht. Die tatsächliche Dynamik wäre zu erkennen, wenn wir in die Nanowelt des Lebendigen „hinein- und wieder heraus-zoomen “: Dabei sind raumzeitliche „Skalensprüngen“ von Picometern $\left(10^{-12}\right)$ zu Metern oder von Femtosekunden (10-15 Sekunden) zu Jahren zu vollziehen, die offenbaren würden, dass die lebenserhaltenden Prozesse nur dank „chaotischer Organisation “ in der Lage sind, sich in einem ständigen „Sturm von Molekülen“ als robust zu erweisen [6]. Zudem würde deutlich, dass sich die „Mechanismen des Lebens “ infolge von Wechselwirkungen ständig verändern. Dies gilt selbst für die heilige Kuh der Präzisionspsychiatrie: die Vorstellung von einem fixen Genom [7].

Zweitens, die Maschinenmetaphorik verstellt den Blick für das heterogene Phänomen der Emergenz. Denn die realen, sich vielfach überschneidenden materiellen Prozesse generieren emergente, qualitativ neue Eigenschaften und Prozesse, die sich nicht durch die Zerlegung mutmaßlicher Aggregate in ihre Bestandteile erhellen lassen [8]. Beispiele hierfür finden sich im Organismus, wohin man schaut: Passive Moleküle werden „aktiv“, wenn sie mit anderen interagieren. Das Zusammenspiel einer Vielzahl toter Moleküle implementiert eine „lebende Zelle“. Einzelne Zellen sind in der Lage, Unterschiede zu detektieren und situationsgemäß variabel zu „agieren“. Komplexe Organismen integrieren vielfältige homöostatische Erfordernisse mit ständig wechselnden Umweltgegebenheiten im Dienste adaptiver „Handlungssteuerung“. Werden die Folgen dieses Wechselspiels von Enterozeption und Exterozeption dank hinreichend entwickelter Nervensysteme z. T. bewusst, so ist von „mentalen
Prozessen“ die Rede. Kurz gesagt: Phänomene wie Selbstorganisation, Leben, Kognition oder Seelenleben - um nur die prominentesten zu nennen - treten in Erscheinung - sie „emergieren“ -, ohne dass fixe, aggregierte Mechanismen dafür verantwortlich wären. Es sind ganz im Gegenteil hochdimensionale, degenerierte Prozesse, die dies bewirken, ohne dafür einen „Master-Mechanismus“ oder einen anthropomorphen „Steuermann“ zu benötigen [9].

Diese systembiologische Kritik des mechanistischen Denkens ist Anlass für grundlegende Überlegungen, von denen ich 2 erwähnen will: Erstens, menschliche Organismen werden stark von kontingenten Entwicklungsbedingungen bestimmt. Eine beachtliche Literatur zum Einfluss nachteiliger Entwicklungsbedingungen - von geburtshilflichen Komplikationen, über ungenügende mütterlicher Sensitivität und traumatischen Erfahrungen bis zum sozioökonomischen Status oder dem Wohnort der Herkunftsfamilie - unterstreicht, dass das pathogene Omics-Geschehen nur als ein diachrones richtig zu verstehen ist. Doch wie soll sich die Geschichte dieser Einflüsse aus einem präzise bestimmten biomolekularen Omics-Befund „hierund-jetzt“ herauslesen lassen? Dies ist nicht möglich, weil lebende Systeme stets in dynamischer Selbstorganisation begriffen sind: Die genannten Entwicklungsbedingungen etwa wirken „multifinal“: dieselbe Bedingung kann unterschiedliche Effekte haben. Und variierende Interaktionen der Elemente können „äquifinale“ Auswirkungen haben: Unterschiedliche Prozesse führen zum selben Ergebnis.

Zweitens, warum betont die Präzisionspsychiatrie die Rolle sub-personaler Omics-Domänen, wohl wissend, dass auch personale und supra-personale Domänen eine wichtige Rolle spielen? Offensichtlich sind ihre „mechanistischen Ambitionen“ Ausdruck einer Denktradition, die die „wesentlichen“ Ursachen auf der „tiefsten“ Ebene bzw. bei den „grundlegenden“ (kleinsten) Elementen sucht. Dabei ist heute klar, dass Lebensprozesse auf zirkulärer, Bottom-up- mit Top-down-Prozessen verbindender Kausalität gründen [9]. Befunde der „social genomics“ etwa belegen, dass soziale Verhältnisse Auswirkungen auf die Genexpression haben [10]. 


\section{Der Organismus als Person}

Personen sind keine geschichtslosen Einzelgänger, sondern Organismen, deren Eigenschaften und Fähigkeiten wesentlich von ihrer sozialen Entwicklungs- und Lerngeschichte bestimmt wird. Ein Blick auf das Verhaltensrepertoire einer kompetenten Person macht klar, wo die Präzisionspsychiatrie zu kurz zu springen droht: Sprachgebrauch, soziale Konventionen und Ontologien, Normen aller Art, Fertigkeiten wie Denken und Entscheiden, Spezialkompetenzen wie Mathematik und enzyklopädisches Wissen etc. sind Ausdruck von Fähigkeiten, die nur mit Hilfe von Anderen erlernt werden können. Es geht um sozial vermittelte Muster kultureller Praxis, die der individuelle Organismus nachbildet, ohne sie selbst definiert bzw. hervorgebracht zu haben. Nur wer lernt, seine Umwelt so wahrzunehmen, zu erkennen, zu benennen und zu handhaben, „wie man dies tut", wird als „psychisch gesund“ gelten, weil er sich in der lebensweltlichen Gemeinschaft adäquat zu bewegen weiß.

Was kennzeichnet Patienten, denen dies nicht angemessen gelingt? Warum tun sich manche Personen schwer, Situationen angemessen zu erkennen, nachvollziehbare Gründen für ihr Tun anzugeben oder ihre Gefühle situationsangemessen zu regulieren? Wer diese Fragen beantworten will, sollte sich vergegenwärtigen, dass es zu den biologischen Besonderheiten unserer Spezies gehört, „sozial geformt“ zu werden. Ohne ein „downstream epistemic engineering “ der nächsten Generation ist die dramatische kulturelle Evolution der letzten 50000 Jahre nicht zu erklären [11]. Eine pathogenetische Pointe davon ist: Je formbarer eine bio-psycho-soziale Entwicklung ist, desto leichter kann sie auch verformt werden. Psychopathologien sind Ausdruck unglücklicher Entwicklungsbedingungen - vom Genom bis zu den sozialen Verhältnissen. Um die rele- vanten kausalen Zusammenhänge einzufangen, müsste die Omics-Hierarchie deshalb um personale und supra-personale Domänen erweitert werden: Auf der personalen Ebene etwa müsste der klinische Phänotyp verhaltensanalytisch erfasst werden, um situationstypische Reaktionsweisen bzw. Defizite des Verhaltensrepertoires zu ermitteln. Da Ausbildung und Aufrechterhaltung dieser Merkmale vom konstitutiven Einfluss supra-personaler Ebenen abhängt - von Beziehungen zu signifikanten Anderen, Partnerschaften, Bezugsgruppen und Gesellschaften -, wären zudem „supra-personale Omics-Domänen“ in den Blick zu nehmen. Ich will damit andeuten, dass eine wahrhaft personalisierte Psychiatrie einem Ideal gleicht, demzufolge alle Parameter, die die Individualität einer Person ausmachen, zu berücksichtigen sind. Da dieses Ideal vermutlich noch lange bloße Utopie bleiben wird, müssen wir uns einstweilen mit „praktischen Rezepten“ bzw. „schlecht replizierbaren Befunden“ zu helfen wissen. Dies ändert jedoch nichts daran, dass Präzision für alle relevanten Domänen zu fordern wäre, um als personalisiertes Vorgehen zu gelten - zumindest aus systembiologischer Sicht. (Dass die personalisierte Psychiatrie auch als „humanistische“ gedacht werden kann, ja sollte, habe ich in diesen Zeilen gar nicht berücksichtigt.)

\section{Interessenkonflikt}

Es bestehen keine Interessenkonflikte.

\section{Korrespondenzadresse}

Dr. Markus R. Pawelzik

EOS Klinik für Psychiatrie

Hammer Str. 18

48153 Münster
Literatur

[1] Aristoteles. Nikomachische Ethik. Berlin: Akademie Verlag; 1956

[2] Wolkenhauer O, Green S, Wellstadt P. A plea for more theory in molecular biology. In: Butcher E, Garry P, Weiss B (eds.) Systems Biology - Applications and Perspectives. Berlin: Springer; 2007

[3] Pawelzik MR. Gibt es psychische Störungen? Journal für Philosophie und Psychiatrie, Mai 2018, original paper

[4] Mias Gl, Snyder M. Personal genomes, quanititative dynamic omics and personalized medicine. Quantitative Biology 2013; 1 (1): 71-90

[5] Cassell EJ. The person in medicine. International Journal of Integrated Care 2010; 10: $50-52$

[6] Hoffmann PM. Life's ratchet: How molecular machines extract order from chaos. New York: Basic Books; 2012

[7] Shapiro JA. Living organisms author their read-write genomes in evolution. Biology 2017; 6: $42 \mathrm{ff}$.

[8] Campbell R. The metaphysics of emergence. London: Palgrave Macmillan; 2015

[9] Noble D. Dance to the tune of life. Biological relativity. Cambridge: Cambridge University Press; 2017

[10] Cole S. Human social genomics. PLOS Genetics 2014; 10 (8): e 1004601

[11] Sterelny K. The evolved apprentice. How evolution made humans unique. Cambridge, Massachusetts: The MIT Press; 2012

Bibliografie

DOI https://doi.org/10.1055/a-0952-6793 Nervenheilkunde 2019; 38: 858-860 (c) Georg Thieme Verlag KG Stuttgart · New York ISSN 0722-1541 